\title{
Density and size of lymphoid follicles are useful clues in differentiating primary intestinal follicular lymphoma from intestinal reactive lymphoid hyperplasia
}

Hsin-Ni Li ${ }^{1}$, Ren Ching Wang ${ }^{1,2}$, Jun-Peng Chen ${ }^{3}$, Sheng-Tsung Chang ${ }^{4,5}$ and Shih-Sung Chuang ${ }^{4,6^{*}}$ (D)

\begin{abstract}
Background: Primary intestinal follicular lymphoma (PI-FL) is a rare and indolent lymphoma and is challenging for diagnosis with endoscopic biopsy specimens. Whole slide imaging (WSI) has been increasingly used for assisting pathologic diagnosis, but not for lymphoma yet, probably because there are usually too many immunostained sections in a single case. In this study we attempted to identify morphological clues of PI-FL in the endoscopic biopsy specimens by measuring various parameters using WSI.
\end{abstract}

Methods: We retrospectively investigated $21 \mathrm{PI-FL}$ cases, and scanned the HE sections from 17 of these cases with endoscopic biopsy specimens. Sections from 17 intestinal biopsies showing reactive lymphoid hyperplasia were scanned for comparison. The density and diameter of lymphoid follicles and the shortest distance of these follicles to the surface epithelia were measured on WSI. Comparisons of the aforementioned parameters were made between the neoplastic and reactive follicles.

Results: The density of follicles was significantly higher in PI-FL than that of reactive hyperplasia (median 0.5 vs. 0.2/ $\mathrm{mm}^{2} ; p<0.01$ ). Furthermore, the neoplastic follicles were significantly larger (median diameter 756.9 vs. $479.7 \mu \mathrm{m}$; $p<0.01)$. The shortest distance of follicles to the surface epithelia tended to be closer in PI-FL (104.7 vs. $177.8 \mu \mathrm{m}$, $p=0.056)$, but not statistically significant.

Conclusions: In this study we found that in PI-FL the density and diameter of lymphoid follicles as measured from WSI were significantly different from that of intestinal reactive lymphoid hyperplasia. When facing the diagnostic challenge between these two entities in routine practice, pathologists might be alerted by these morphological clues and request for immunohistochemistry for differential diagnosis.

Keywords: Digital pathology, Duodenal-type follicular lymphoma, Follicular lymphoma, Intestinal lymphoma, Lymphoid hyperplasia, Whole slide imaging

\footnotetext{
* Correspondence: cmh5301@mail.chimei.org.tw

${ }^{4}$ Department of Pathology, Chi-Mei Medical Center, 901 Chung-Hwa Road, Yong-Kang District, Tainan 71004, Taiwan

${ }^{6}$ Department of Pathology, School of Medicine, College of Medicine, National Taiwan University, Taipei, Taiwan

Full list of author information is available at the end of the article
}

(C) The Author(s). 2020 Open Access This article is licensed under a Creative Commons Attribution 4.0 International License, which permits use, sharing, adaptation, distribution and reproduction in any medium or format, as long as you give appropriate credit to the original author(s) and the source, provide a link to the Creative Commons licence, and indicate if changes were made. The images or other third party material in this article are included in the article's Creative Commons licence, unless indicated otherwise in a credit line to the material. If material is not included in the article's Creative Commons licence and your intended use is not permitted by statutory regulation or exceeds the permitted use, you will need to obtain permission directly from the copyright holder. To view a copy of this licence, visit http://creativecommons.org/licenses/by/4.0/ The Creative Commons Public Domain Dedication waiver (http://creativecommons.org/publicdomain/zero/1.0/) applies to the data made available in this article, unless otherwise stated in a credit line to the data. 


\section{Background}

Whole slide imaging (WSI), the scanning of conventional glass slides as digital files, has been increasingly employed by pathologists for assisting diagnosis, education, and research purposes. Recently several studies have validated the accuracy of WSI in pathological diagnosis, suggesting that WSI can be used for routine practice, which has been realized in a Dutch academic pathology laboratory where the pathological diagnosis is fully digitalized [1-4]. The concordance rates in diagnosis between using conventional light microscope and WSI range from 87 to $96 \%$, depending on various subspecialties [1, 5-9]. However, most of previously published studies mainly targeted small specimens and excluded cases requiring immunohistochemistry, such as lymphoma cases. So far there is only one published report investigating the concordance rate between digital pathology and conventional microscopic histopathology and the researchers found that WSI was a reliable technology for lymphoma diagnosis [10].

Follicular lymphoma (FL) is one of the most common low-grade B cell lymphomas. FL is a heterogeneous disease, and may be further divided into many different subtypes in terms of age of onset, involved organs and genetic abnormality. In the revised fourth edition of World Health Organization (WHO) classification of tumors of hematopoietic and lymphoid tissues, primary intestinal FL (PI-FL) is listed as a rare and specific variant of FL with distinct clinical and biological features [11]. PI-FL predominantly occurs in the second portion of duodenum as multiple whitish polypoid nodules [12, 13]. Unlike patients with systemic nodal FL, most patients with PI-FL have localized diseases and excellent prognosis even without treatment [11-13]. However, long-term follow-up is recommended as rare cases with histological transformation to high-grade lymphoma have been reported [14, 15]. Considering the uniqueness of PI-FL, thorough clinical, laboratory, endoscopic and radiologic workups to differentiate PI-FL from systemic nodal FL with secondary gastrointestinal (GI) tract involvement have been suggested [16].

PI-FL shares the same histopathology, immunophenotype, and the hallmark $t(14 ; 18)$ translocation with systemic nodal FL $[12,17]$. On the other hand, expression of certain markers, such as memory B-cell marker CD27, molecules related to B-cell homing such as chemokine (C-C motif) ligand 20 (CCL20) and mucosal vascular addressin cell adhesion molecule 1 (MAdCAM-1), lack of activation-induced cytidine deaminase (AID), and variable immunoglobulin heavy chain gene $(V H)$ usage suggest a genetic similarity of PI-FL with gastric mucosa-associated lymphoid tissue lymphoma, but different from systemic nodal FL [13, 17-20]. In addition, follicular dendritic cells (FDC) residing in the periphery of neoplastic follicles is more frequent in PI-FL cases as compared to nodal FLs, in which the FDC usually form dense meshworks. Takata $\mathrm{K}$. et al. thus suggested that recognizing different FDC patterns is useful for differentiating PI-FL from secondary GI involvement by systemic FL [13].

It is important to make an accurate diagnosis by endoscopic biopsy specimens to facilitate appropriate management. In this study we retrospectively analyzed PI-FL cases in an attempt to identify morphological features of PI-FL by measuring various parameters on WSI. These morphological clues might be useful in differentiating reactive lymphoid hyperplasia from PI-FL in endoscopic biopsy specimens.

\section{Methods \\ Patients}

We searched our databases of the Department of Pathology and Laboratory Medicine at the Taichung Veterans General Hospital, Taichung, and Chi-Mei Medical Center, Tainan, Taiwan for PI-FL from January 2001 to December 2018. Cases of systemic nodal FL with secondary GI tract involvement were excluded after clinical investigation and imaging studies.

We reviewed medical records for clinical information, including age, gender, anatomical sites, clinical symptoms, endoscopic findings, sampling methods, and staging results according to the Lugano Classification. The Follicular Lymphoma International Prognostic Index (FLIPI) was used for the evaluation of disease status for prognosis [21]. Follow-up period was measured from the date of diagnosis to the date of last follow-up. For comparison, 17 endoscopic biopsy specimens from the corresponding GI sites diagnosed as reactive follicular hyperplasia were recruited as controls.

\section{Histopathologic and immunohistochemical analyses}

The cases diagnosed as FL were confirmed by three experienced hematopathologists (RCW, STC and SSC) according to the $2017 \mathrm{WHO}$ criteria. Two of us (HNL and $\mathrm{RCW}$ ) confirmed the diagnosis of reactive hyperplasia of the control cases.

Supplementary Table 1 lists the antibodies used and the staining condition for immunohistochemistry. The staining pattern of FDC meshworks was classified either as nodal type/pattern (>30\% positive FDC cells with FDC mainly located in the center of the follicles) or duodenal type/pattern $(<5 \%$ positive FDC cells with FDC in the periphery of the follicles), as previously defined by Takata K. et al. [22]

\section{Interphase fluorescence in situ hybridization (FISH)}

Locus-specific interphase FISH was performed on paraffin-embedded tissue sections of $4 \mu$ thickness as previously described [23, 24]. In brief, de-paraffinized sections were pre-treated by pressure-cooking for $10 \mathrm{~min}$ 
in Milli-Q water and subsequent incubation in pepsin solution for $25 \mathrm{~min}$ at $37^{\circ} \mathrm{C}$ to increase DNA accessibility. Sections were then dehydrated through ethanol and air-dried. The appropriate probe mix $(1.0 \mu \mathrm{l})$ was applied to the tissue section and covered with a round $8 \mathrm{~mm}$ cover slip. Both probe and target DNA were simultaneously denatured at $73{ }^{\circ} \mathrm{C}$ for $5 \mathrm{~min}$ and incubated up to 2 days at $45^{\circ} \mathrm{C}$. Posthybridisation washes were performed according to the "rapid-wash protocol" provided by Vysis, Downers Grove, IL. Sections were counterstained with 4,6-diamidino-2-phenylindole (DAPI) and mounted in Vectashield antifade solution (Vector Laboratories, Burlingame, CA, USA).

Dual Color Break Apart Rearrangement probes for IGH and $B C L 2$ and Dual Color Dual Fusion Translocation probe for $I G H / B C L 2$ reciprocal translocation (Vysis/Abbott Laboratories Ltd., Maidenhead, UK.) were used.

\section{WSI and measurements}

Hematoxylin and eosin-stained biopsy sections were scanned at 400x magnification (3D Histech Pannoramic 250, Budapest, Hungary). For each biopsy, the total surface area of the biopsy specimen was measured and the total number of follicles counted. For those specimens containing more than one fragment, the total number of follicles and the total areas of all fragments were respectively added up. We defined the density of follicles by dividing the number of lymphoid follicles by the total surface area of the biopsy specimen. The diameter (the greatest dimension) of a follicle (including the mantle zones and the follicular centers) and the shortest distance of that particular follicle to the surface epithelia were measured. Figure 1 depicts a representative example of various measurements using WSI. In this study we did not scan sections from resection specimens as the large number of follicles in such specimens may skew the data of endoscopic biopsies.

\section{Statistical analysis}

The density and size of follicles and the shortest distance of the follicles to the surface epithelia were compared between the study and control groups. We used MannWhitney $U$ test to obtain median values. Data analyses
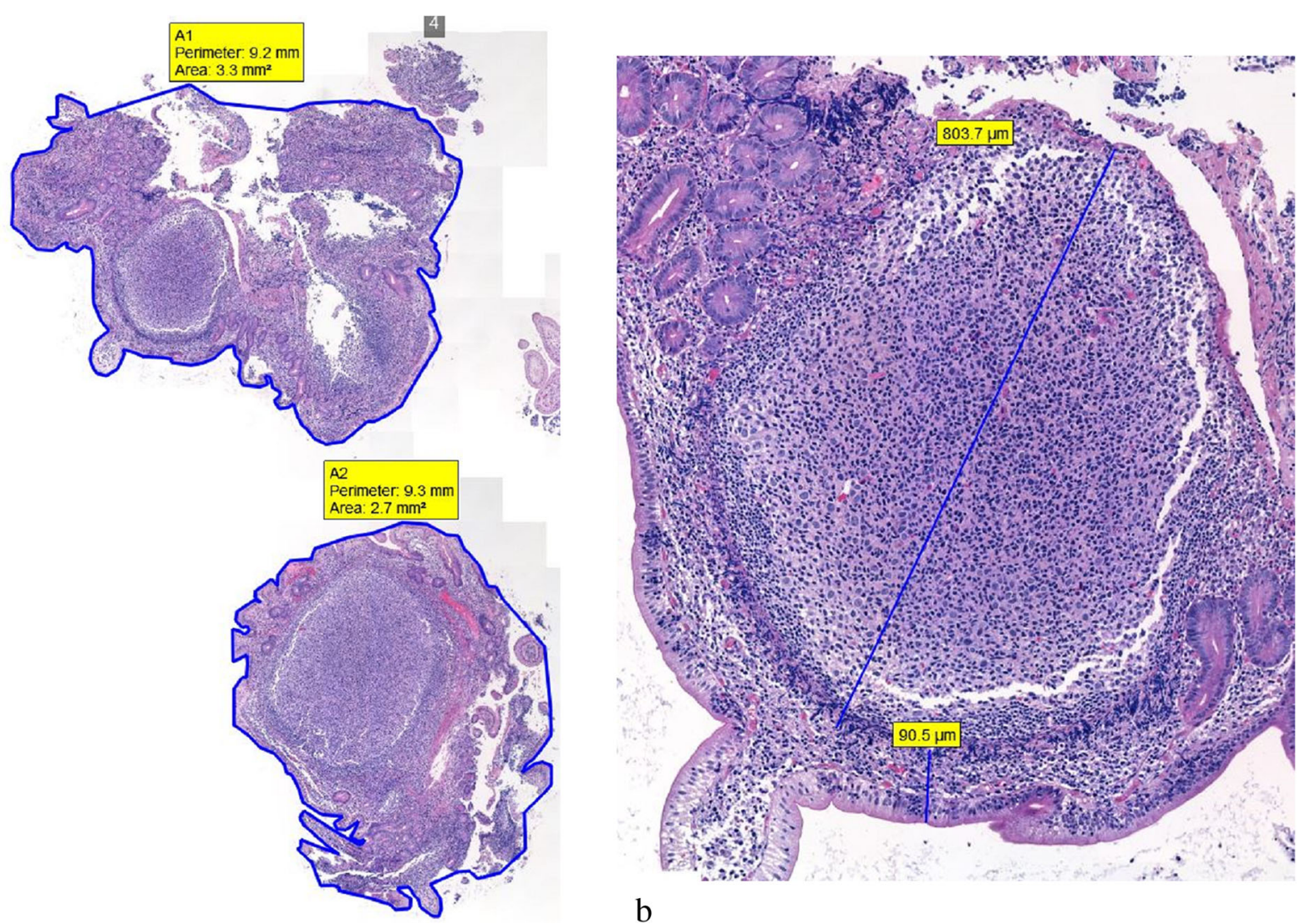

b

Fig. 1 A representative example (Case no. 3) of using whole slide imaging in measuring various parameters. a, The density (D) of follicles is measured by dividing the number of lymphoid follicles by the total surface area of the biopsy specimen (with summation of the measurements from all the tissue fragments; $D=$ number of follicles/area; in this case, $2 /(3.3+2.7)=0.3333)$. $\mathbf{b}$, This panel shows the diameter (the greatest dimension) of a follicle $(803.7 \mu \mathrm{m})$ and the nearest distance of this follicle to the surface epithelia $(90.5 \mu \mathrm{m})$ 
were performed using the Statistical Package for the Social Science (IBM SPSS version 22.0; International Business Machines Corp, New York, USA). Differences were considered significant when $P<0.05$.

\section{Results}

\section{Clinical features of the patients with PI-FL}

Supplementary Table 2 lists the pertinent clinicopathological findings and follow-up information of the patients with PI-FL, while Table 1 summarizes the various clinical features of the PI-FL cases and the control cases with reactive hyperplasia. Eleven PI-FL patients were from Taichung Veterans General Hospital and ten patients were from Chi-Mei Medical Center. One of the cases from Chi-Mei Medical Center has been reported previously [25]. There was no difference in terms of age, gender, and biopsied sites between the patients with PI$\mathrm{FL}$ and the controls.

Of the PI-FL cases, there were 10 males and $11 \mathrm{fe}$ males with a median age of 61 (range, 24-90). These diseases were detected either at routine medical checkup with endoscopy $(n=8)$ or when the patients sought medical advice with symptoms including epigastric discomfort $(n=5)$, stool occult blood $(n=2)$, bowel habit change $(n=2)$, abdominal pain $(n=1)$, recurrent postprandial hiccup $(\mathrm{n}=1)$ and small bowel obstruction $(\mathrm{n}=$ $1)$. The most common primary site was the duodenum ( $n=11 ; 52 \%)$, followed by the ileocecum $(n=6 ; 29 \%)$, jejunum $(\mathrm{n}=2 ; 10 \%)$ and multiple foci in the GI tract $(\mathrm{n}=$ $2 ; 10 \%)$. The endoscopic findings showed polypoid or nodular lesions in the presence or absence of ulceration, whitish plaques or hyperemia. Most cases $(n=16 ; 76 \%)$ were from endoscopic biopsies; with the remaining one (5\%) from mucosal resection and four (19\%) from bowel resection. Excluding the three cases with incomplete staging, the patients were either at stage I $(n=13)$ or II $(n=5)$. As shown in Table 1 , around half of patients $(n=9)$ were followed without treatment (watch-andwait), while the other half $(n=10)$ received localized radiotherapy or chemotherapy. No patient died of the disease in a median follow-up time of 41 months (range, 12-208).

\section{Histopathologic and immunohistochemical findings}

Table 2 summarizes the histological features and the immunohistochemical results. Microscopically, the neoplastic follicles were located in the lamina propria (Fig. 2a) with occasional surface erosion or ulceration. They were relatively round-shaped with absent or attenuated mantles and absence of polarity (Fig. 2a). Under high-power examination, the follicular center cells were monotonous comprising small- to medium-sized centrocytes without tangible body macrophages (Fig. 2b). All 21 cases were classified as low-grade FL.
Table 1 Pertinent clinical information of the patients with PI-FL and the control cases with reactive lymphoid hyperplasia

\begin{tabular}{|c|c|c|}
\hline Parameters & $\mathrm{PI}-\mathrm{FL}$ & Control \\
\hline Case number & $n=21$ & $n=17$ \\
\hline \multicolumn{3}{|l|}{ Age (years) } \\
\hline Range & $24-90$ & $29-65$ \\
\hline Median & 61 & 54 \\
\hline \multicolumn{3}{|l|}{ Gender } \\
\hline Male/Female & $10 / 11$ & $8 / 9$ \\
\hline \multicolumn{3}{|l|}{ Symptoms } \\
\hline None & 8 & 15 \\
\hline Epigastric discomfort & 4 & 2 \\
\hline Stool occult blood & 2 & 0 \\
\hline Bowel habit change & 1 & 0 \\
\hline Epigastric discomfort and bowel habit change & 1 & 0 \\
\hline Abdominal pain & 1 & 0 \\
\hline Recurrent post-prandial hiccup & 1 & 0 \\
\hline Small bowel obstruction & 1 & 0 \\
\hline NA & 2 & 0 \\
\hline \multicolumn{3}{|l|}{ Site } \\
\hline Duodenum & $11(52 \%)$ & 11 \\
\hline Ileocecum & $6(29 \%)$ & 4 \\
\hline Jejunum & $2(10 \%)$ & 0 \\
\hline Colon, multiple & $2(10 \%)$ & 2 \\
\hline \multicolumn{3}{|l|}{ Endoscopic findings } \\
\hline Polypoid lesions & 10 & 2 \\
\hline Whitish plaques & 3 & 0 \\
\hline Nodularity & 2 & 1 \\
\hline Hyperemia & 1 & 6 \\
\hline Bloody fluid & 1 & 0 \\
\hline NA & 4 & 8 \\
\hline \multicolumn{3}{|l|}{ Sampling method } \\
\hline Biopsy/Mucosal resection/Resection & $16 / 1 / 4$ & $17 / 0 / 0$ \\
\hline \multicolumn{3}{|l|}{ Stage } \\
\hline I/II/NA & $13 / 5 / 3$ & \\
\hline \multicolumn{3}{|l|}{ FLIPI } \\
\hline $0 / 1 / 2 / 3 / N A$ & $7 / 7 / 2 / 2 / 3$ & \\
\hline \multicolumn{3}{|l|}{ Treatment } \\
\hline None/CT/RT/NA & $9 / 8 / 2 / 2$ & \\
\hline \multicolumn{3}{|l|}{ Follow-up time (months) } \\
\hline Range & $12-208$ & \\
\hline Median & 41 & \\
\hline
\end{tabular}

Abbreviation: CT Chemotherapy, NA Not available, PI-FL Primary intestinal follicular lymphoma, $R T$ Radiotherapy 
Table 2 Histopathologic, immunohistochemical and FISH findings of PI-FL and control cases with reactive lymphoid hyperplasia

\begin{tabular}{|c|c|c|}
\hline Parameters & PI-FL $(n=21)$ & Control $(n=17)$ \\
\hline \multicolumn{3}{|l|}{ Features of the follicles } \\
\hline Polarity & Lost & Preserved \\
\hline Cellular components & Mainly centrocytes & Mixed populations \\
\hline Tingible body macrophages & Absent & Present \\
\hline \multicolumn{3}{|l|}{ Histologic grade } \\
\hline $1 / 2$ & $19(91 \%) / 2(10 \%)$ & NA \\
\hline \multicolumn{3}{|l|}{ Immunohistochemistry } \\
\hline CD10 expression & $21(100 \%)$ & \\
\hline BCL6 expression & $21(100 \%)$ & \\
\hline BCL2 expression & $21(100 \%)$ & \\
\hline FDC pattern & & Tight meshwork \\
\hline Duodenal & 14 (67\%; 11 duodenum, 1 ileum and 2 colon) & \\
\hline Nodal & 6 (29\%; 4 ileocecum and 2 jejunum) & \\
\hline Mixed duodenal and nodal & $1(5 \%)$ & \\
\hline \multicolumn{3}{|l|}{$\mathrm{FISH} / G H / B C L 2$} \\
\hline Positive/ Negative/ Failed/ Not tested & $17 / 1 / 2 / 1$ & \\
\hline
\end{tabular}

Abbreviation: FDC Follicular dendritic cell, FISH Fluorescence in situ hybridization for reciprocal translocation of IGH and BCL2 loci, PI-FL Primary intestinal follicular lymphoma

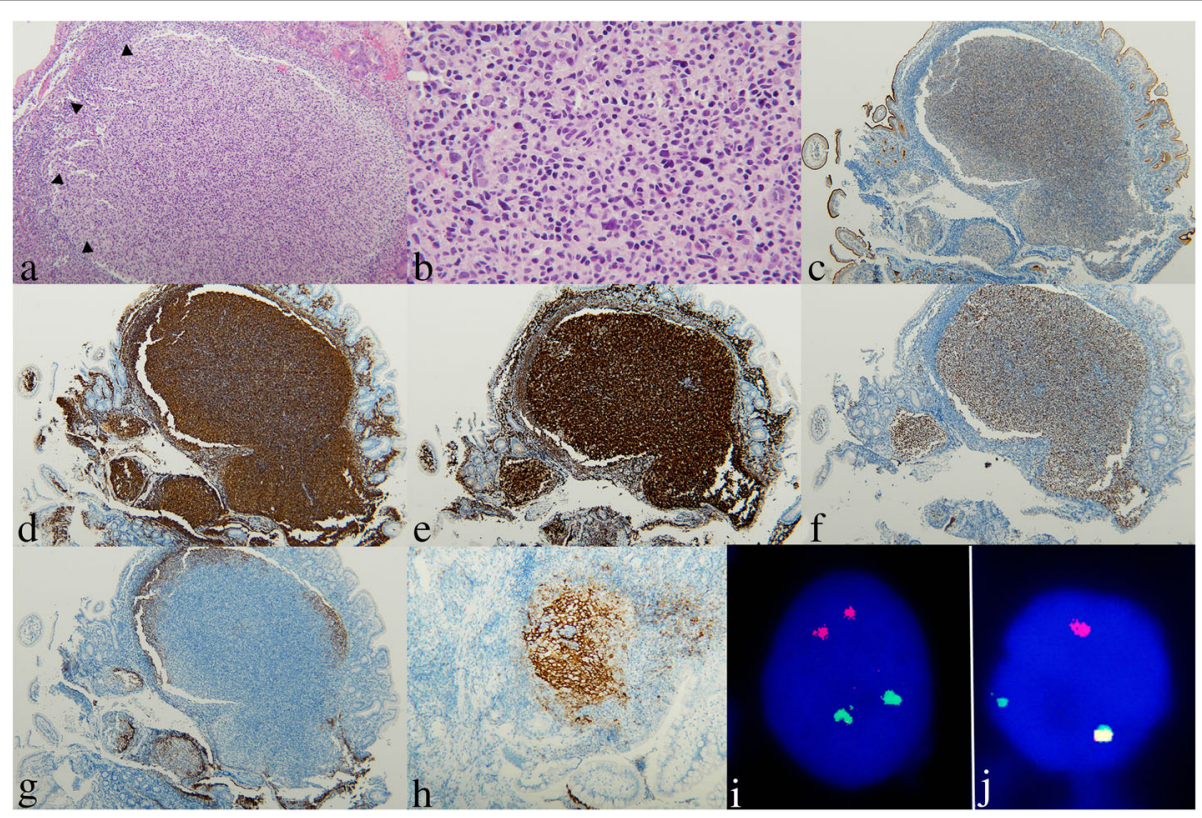

Fig. 2 A representative case of a low-grade primary intestinal follicular lymphoma (Case No. 4, except Panel h, i and j). a Low-power view shows a large lymphoid follicle in the lamina propria of duodenal mucosa (H\&E stain, $\times 40$ ). The mantle zone indicated by black arrowheads is attenuated. In this case, the mucosa is intact, without erosion or ulceration. $\mathbf{b}$ The follicle is composed mainly of small- to medium-sized centrocytes without tingible body macrophages $(\times 400)$. Immunohistochemistry shows that the neoplastic cells express CD10 (c, $\times 40)$, CD20 (d, $\times 40), B C L-2(\mathbf{e}, \times 40)$, and BCL-6 $(\mathbf{f}, \times 40)$. The follicular dendritic meshworks of this case show a characteristic duodenal pattern by immunostaining with CD23 $(\mathbf{g}, \times 40)$, in contrast to the nodal pattern in Case no. $11(\mathbf{h}, \times 40)$. FISH assay using Vysis /GH/BCL2 Dual Color, Dual Fusion Translocation Probe (IGH in SpectrumGreen and BCL2 in SpectrumOrange). i A normal cell showing two orange and two green signals (2O2G), indicating no translocation of either IGH or BCL2 genes. $\mathbf{j}$ A neoplastic cell with reciprocal IGH/BCL2 translocation showing one orange, one green (representing the normal homolog) and one fused (orange/green) signal in yellow (1O1G1F; i and j from Case no. 21) 
The neoplastic cells in all cases expressed CD20 (Fig. 2d) and BCL2 (Fig. 2e), with extrafollicular spreading to the overlying villi. The neoplastic follicular center cells of all cases expressed CD10 (Fig. 2c) and BCL-6 (Fig. 2f). FDC markers (CD21, CD23, or CD35) revealed a "duodenal pattern" in 14 cases, in which the FDC meshworks formed a condensed rim of enhanced staining at the periphery of the follicles but sparing the central area (Fig. $2 \mathrm{~g})$. The tumors of these 14 cases were located in the duodenum $(n=11)$, ileum $(\mathrm{n}=1)$, and colon $(n=2)$. In six patients, the FDC pattern was nodal (Fig. $2 \mathrm{~h}$ ), and all these tumors were located in non-duodenal sites including ileocecum $(n=4)$ and jejunum $(\mathrm{n}=2)$. Interestingly, in one case the FDC pattern in the initial biopsy was of duodenal type, while that of the resection specimen was of nodal type. Taken together, in our cases when the PIFLs were located in the duodenum or other GI sites, FDC meshwork usually appeared as duodenal pattern; for those tumors occurring in the non-duodenal sites, the FDC meshworks tended to of nodal pattern. Coexisting duodenal and nodal patterns also occurred.

\section{Interphase FISH}

FISH assay was successful in 18 cases. IGH and BCL2 loci were rearranged in $94 \%(17 / 18)$ and $83 \%(15 / 18)$ cases, respectively. Seventeen (94\%) cases showed IGH/ $B C L 2$ reciprocal translocation.

\section{WSI analysis}

Table 3 summaries the results of measurements using WSI between neoplastic follicles in PI-FL and control cases with reactive lymphoid hyperplasia. We found that the density of follicles was higher in PI-FL than that of reactive hyperplasia (median $0.5 / \mathrm{mm}^{2}$ vs. $0.2 / \mathrm{mm}^{2} ; p<$ $0.01)$ and that the neoplastic follicles were significantly larger (median diameter $756.9 \mu \mathrm{m}$ vs. $479.7 \mu \mathrm{m} ; p<$ 0.01 ). The shortest distance of follicles from the surface epithelia was of no statistical significance between these two groups $(104.7 \mu \mathrm{m}$ vs. $177.8 \mu \mathrm{m} ; p=0.056)$, although that of the PI-FL cases tended to be closer to the mucosal surface.

\section{Discussion}

Although rare, PI-FL has been characterized recently, particularly the duodenal-type FL. Surgical specimens are easier for diagnosis; however, with increasing use of endoscopy, accurate pathologic diagnosis on small biopsy specimens remains challenging. In this current study of PI-FL with WSI, we found that the average density and diameter of neoplastic follicles were larger than those of reactive lymphoid hyperplasia in the GI tract. Interestingly, in contrast to the study by Crocker et al in 1983, in which they used image analyzer and found that reactive lymph nodes had larger mean follicle areas than nodal FL [26]. The discrepancy between their and our studies may come from the different types of specimens/organs used for analysis, that is, large nodal tissue vs. endoscopic intestinal biopsy specimens. In addition, the neoplastic follicles in our PI-FL samples tended to be located more superficially, although not statistically significant. Occasional ulcerations or erosions of the surface mucosa were noted as well. Thus, we suggest that when encountering endoscopic biopsies with the presence of superficially located large follicles and a high density of follicles, immunohistochemistry is mandatory to exclude the possibility of PI-FL. Further WSI studies might include cases of systemic nodal FL with secondary involvement of GI tract, to compare the histologic parameters of neoplastic follicles between primary and secondary FL.

In keeping with other series of PI-FL [27, 28], the majority of our patients were middle-aged or elderly. Onethird of our patients were asymptomatic and the remaining patients presented with ambiguous abdominal discomfort or bloody stools. In the literature, most of the cases were detected by endoscopy with typical polypoid lesions in the duodenum (65\%), followed by jejunum and ileum (20\%) [13]. Our cases showed a similar trend with duodenum as the most common site $(11 / 21 ; 52 \%)$, followed by ileocecum and jejunum $(8 / 21 ; 38 \%)$. In the largest series of PI-FL so far in the literature, Schmatz et al reported that in 11 (17\%) of 63 patients another segment of the small bowel (i.e., jejunum and/or ileum) was involved [12]. In our study, in most of the cases (19/ 21; 91\%), the tumors were located only in a single anatomic site. Primary FL at different GI sites has been reported to possess different endoscopic features as visualized by more advanced and detailed endoscopic examination. For example, the most frequent endoscope finding in the stomach was ulcerated submucosal tumor while small granular lesions were the predominant findings in the duodenum [29-31]. All of our cases showed

Table 3 Comparisons between neoplastic follicles in PI-FL and control cases with intestinal reactive lymphoid hyperplasia

\begin{tabular}{llll}
\hline & PI-FL $(n=17)$ & Control $(n=17)$ & $p$ value \\
\hline Diameter $(\mu \mathrm{m})$ & $756.9(576.8-929.9)$ & $479.7(337.1-607.2)$ & $0.001^{* *}$ \\
Distance from surface epithelia $(\mu \mathrm{m})$ & $104.7(75.9-194.9)$ & $177.8(99.2-302.9)$ & 0.056 \\
Density (No./Surface area) & $0.5(0.3-0.7)$ & $0.2(0.1-0.3)$ & $0.004^{* *}$ \\
\hline
\end{tabular}

Mann-Whitney U test. Median (Q1-Q3). ${ }^{*} p<0.05,{ }^{* *} p<0.01$

Abbreviation: No. Number of follicles 
low clinical stage without mortality or high-grade transformation during the follow-up period. Application of the FLIPI developed for nodal FL seemed irrelevant to the prognosis of our patients. However, data from more cases should be collected to investigate the feasibility of using FLIPI in PI-FL.

Immunophenotypically, the neoplastic follicular center cells expressed germinal center cell markers CD10 and BCL-6, with aberrant expression of BCL-2 due to $t(14$; 18)/IGH-BCL2 translocation. In our current study, all PI-FL cases demonstrated BCL-2 protein expression and all cases expressed CD10 and BCL-6. The characteristic duodenal pattern of FDC meshworks was observed in all of our duodenal examples of PI-FL, while those in the non-duodenal sites had either nodal or duodenal patterns. The phenomenon was also observed in a previous study [22]. Interestingly, one of our cases had FDC meshworks in a mixed nodal and duodenal pattern. To our knowledge, to date there are no data suggesting PIFLs at different GI sites might have different prognosis. The significance of FDC patterns has not yet been fully investigated either.

Using FISH, we confirmed IGH/BCL2 translocation in most (94\%) of the successfully assayed cases, while the neoplastic cells in all cases expressed BCL-2 protein. The hallmark translocation $\mathrm{t}(14 ; 18)(\mathrm{q} 32 ; \mathrm{q} 21)$ was detected in around $85 \%$ of PI-FL by FISH [12, 22], similar to that of nodal FL. Although in our previous study we found a relatively lower frequency $(63 \%)$ of $t(14 ; 18)$ in low-grade FL in Taiwan [23]; in the current study we found that PI-FL in Taiwan carried a similar rate of $t(14$; 18 ) as that of the Western nodal FL and Japanese PI-FL. The prognostic significance of $t(14 ; 18)$ in patients with PI-FL has not been delineated yet. Studies on larger numbers of cases are warranted.

\section{Conclusions}

The incidence of PI-FL might be underestimated as the patients might be asymptomatic or might have ambiguous symptoms, as exemplified in our study that eight (38\%) patients were discovered at health examination with endoscopy. Although the case number is limited, our study, so-far the largest from Taiwan, showed histologic and clinical features characteristic of PI-FL. Most importantly, our study is the first to utilize digital imaging technique to assess the histologic parameters of PI-FL vs. intestinal reactive lymphoid hyperplasia. Our results indicate that superficially located large follicles and a high density of follicles in endoscopic biopsy specimens are surrogates for PI-FL and immunohistochemistry is mandatory to confirm the diagnosis. Larger series of studies are required to better characterize and to verify the parameters measured from WSI and their usefulness as adjuncts for pathological diagnosis.

\section{Supplementary information}

Supplementary information accompanies this paper at https://doi.org/10. 1186/s13000-020-00991-3.

Additional file 1: Table S1. Antibodies used and the immunostaining conditions for this study. Table S2. Pertinent clinicopathological findings and follow-up information of the patients with primary intestinal follicular lymphoma.

\section{Abbreviations}

FDC: Follicular dendritic cell; FISH: Fluorescence in situ hybridization; FL: Follicular lymphoma; FLIPI: Follicular Lymphoma International Prognostic Index; Gl: Gastrointestinal; PI-FL: Primary intestinal follicular lymphoma; WHO: World Health Organization; WSI: Whole slide imaging

\section{Acknowledgments}

The authors thank Ms. Jing-Yi Lai, Department of Pathology and Laboratory Medicine of Taichung Veterans General Hospital for the assistance in slide scanning and data extraction.

\section{Authors' contributions}

HNL and SSC designed the study. RCW, STC and SSC investigated and provided the study cases. HNL performed the experiments. All authors analyzed the data. HNL and SSC wrote the manuscript. SSC revised and edited the manuscript. The authors read and approved the final manuscript.

Funding

This study was supported by a research grant from the Ministry of Science and Technology, Taipei, Taiwan (to SSC).

Availability of data and materials

All data are available from the first author upon request.

Ethics approval and consent to participate

The Internal Review Board approved this study.

Consent for publication

Not applicable.

Competing interests

None declared.

\section{Author details}

${ }^{1}$ Department of Pathology and Laboratory Medicine, Taichung Veterans General Hospital, Taichung, Taiwan. ${ }^{2}$ Department of Nursing, HungKuang University, Taichung, Taiwan. ${ }^{3}$ Biostatistics Task Force, Taichung Veterans General Hospital, Taichung, Taiwan. ${ }^{4}$ Department of Pathology, Chi-Mei Medical Center, 901 Chung-Hwa Road, Yong-Kang District, Tainan 71004, Taiwan. ${ }^{5}$ Department of Nursing, National Tainan Institute of Nursing, Tainan, Taiwan. ${ }^{6}$ Department of Pathology, School of Medicine, College of Medicine, National Taiwan University, Taipei, Taiwan.

Received: 11 February 2020 Accepted: 17 June 2020

Published online: 07 July 2020

\section{References}

1. Tabata K, Mori I, Sasaki T, et al. Whole-slide imaging at primary pathological diagnosis: validation of whole-slide imaging-based primary pathological diagnosis at twelve Japanese academic institutes. Pathol Int. 2017;67(11): 547-54

2. Bauer TW, Schoenfield L, Slaw RJ, Yerian L, Sun Z, Henricks WH. Validation of whole slide imaging for primary diagnosis in surgical pathology. Arch Pathol Lab Med. 2013;137(4):518-24.

3. Campbell WS, Lele SM, West WW, Lazenby AJ, Smith LM, Hinrichs SH. Concordance between whole-slide imaging and light microscopy for routine surgical pathology. Hum Pathol. 2012;43(10):1739-44.

4. Stathonikos N, Nguyen TQ, Spoto CP, Verdaasdonk MAM, van Diest PJ. Being fully digital: perspective of a Dutch academic pathology laboratory. Histopathology. 2019;75(5):621-35. 
5. Saco A, Diaz A, Hernandez M, et al. Validation of whole-slide imaging in the primary diagnosis of liver biopsies in a university hospital. Dig Liver Dis. 2017:49(11):1240-6.

6. Ordi J, Castillo P, Saco A, et al. Validation of whole slide imaging in the primary diagnosis of gynaecological pathology in a university hospital. J Clin Pathol. 2015:68(1):33-9.

7. Al-Janabi S, Huisman A, Jonges GN, Ten Kate FJ, Goldschmeding R, van Diest PJ. Whole slide images for primary diagnostics of urinary system pathology: a feasibility study. J Renal Inj Prev. 2014;3(4):91-6.

8. Al-Janabi S, Huisman A, Willems SM, Van Diest PJ. Digital slide images for primary diagnostics in breast pathology: a feasibility study. Hum Pathol. 2012:43(12):2318-25

9. Al-Janabi S, Huisman A, Vink A, et al. Whole slide images for primary diagnostics of gastrointestinal tract pathology: a feasibility study. Hum Pathol. 2012:43(5):702-7.

10. Amin S, Mori T, Itoh T. A validation study of whole slide imaging for primary diagnosis of lymphoma. Pathol Int. 2019;69(6):341-9.

11. Swerdlow SHCE, Harris NL, Jaffe ES, Pileri SA, Stein H, Thiele J. WHO classification of Tumours of Haematopoietic and lymphoid tissues. . Revised 4th ed; 2017. IARC; No. 2.

12. Schmatz Al, Streubel B, Kretschmer-Chott E, et al. Primary follicular lymphoma of the duodenum is a distinct mucosal/submucosal variant of follicular lymphoma: a retrospective study of 63 cases. J Clin Oncol. 2011; 29(11):1445-51.

13. Takata K, Miyata-Takata T, Sato Y, et al. Gastrointestinal follicular lymphoma: current knowledge and future challenges. Pathol Int. 2018;68(1):1-6.

14. Hangai S, Nakamura F, Kamikubo Y, et al. Primary gastrointestinal follicular lymphoma with histological transformation. Ann Hematol. 2013;92(7):993-4.

15. Tanigawa T, Abe R, Kato J, et al. Histological transformation in duodenaltype follicular lymphoma: a case report and review of the literature. Oncotarget. 2019;10(36):3424-9.

16. Iwamuro $\mathrm{M}$, Kondo $\mathrm{E}$, Takata $\mathrm{K}$, Yoshino $\mathrm{T}$, Okada $\mathrm{H}$. Diagnosis of follicular lymphoma of the gastrointestinal tract: a better initial diagnostic workup. World J Gastroenterol. 2016;22(4):1674-83.

17. Takata K, Sato Y, Nakamura N, et al. Duodenal and nodal follicular lymphomas are distinct: the former lacks activation-induced cytidine deaminase and follicular dendritic cells despite ongoing somatic hypermutations. Mod Pathol. 2009;22(7):940-9.

18. Takata K, Tanino M, Ennishi D, et al. Duodenal follicular lymphoma: comprehensive gene expression analysis with insights into pathogenesis. Cancer Sci. 2014;105(5):608-15.

19. Sato $Y$, Ichimura $K$, Tanaka $T$, et al. Duodenal follicular lymphomas share common characteristics with mucosa-associated lymphoid tissue Iymphomas. J Clin Pathol. 2008;61(3):377-81.

20. Sato Y, Nakamura N, Nakamura S, et al. Deviated VH4 immunoglobulin gene usage is found among thyroid mucosa-associated lymphoid tissue lymphomas, similar to the usage at other sites, but is not found in thyroid diffuse large B-cell lymphomas. Mod Pathol. 2006;19(12):1578-84.

21. Solal-Celigny $P$, Roy $P$, Colombat $P$, et al. Follicular lymphoma international prognostic index. Blood. 2004;104(5):1258-65.

22. Takata K, Sato Y, Nakamura N, et al. Duodenal follicular lymphoma lacks AID but expresses BACH2 and has memory B-cell characteristics. Mod Pathol. 2013;26(1):22-31

23. Chang ST, Lu YH, Lu CL, et al. Follicular lymphoma in Taiwan: a low frequency of $t(14 ; 18)$, with grade $3 A$ tumours more closely related to grade 3B than to low-grade tumours. Histopathology. 2013;63(1):1-12.

24. Chuang SS, Ye H, Yang SF, et al. Perforation predicts poor prognosis in patients with primary intestinal diffuse large B-cell lymphoma. Histopathology. 2008;53(4):432-40.

25. Chuang SS, Chang ST, Liu H, et al. Intramucosal follicular lymphoma in the terminal ileum with nodal involvement. Pathol Res Pract. 2007;203(9):691-4.

26. Crocker J, Jones EL, Curran RC. A quantitative study of the size of benign and malignant lymphoid follicles. J Clin Pathol. 1983;36(9):1055-61.

27. Takata K, Okada H, Ohmiya N, et al. Primary gastrointestinal follicular lymphoma involving the duodenal second portion is a distinct entity: a multicenter, retrospective analysis in Japan. Cancer Sci. 2011;102(8):1532-6.

28. Yoshino T, Miyake $K$, Ichimura $K$, et al. Increased incidence of follicular lymphoma in the duodenum. Am J Surg Pathol. 2000;24(5):688-93.

29. Iwamuro M, Okada H, Takata K, et al. Magnifying endoscopic observation of duodenal involvement of follicular lymphoma before and after chemotherapy. Intern Med. 2015;54(14):1741-5.
30. Iwamuro M, Okada H, Takata K, et al. Diagnostic accuracy of endoscopic biopsies for the diagnosis of gastrointestinal follicular lymphoma: a clinicopathologic study of 48 patients. Ann Diagn Pathol. 2014;18(2):99-103.

31. Maeshima AM, Taniguchi $H$, Suzuki $T$, et al. Comparison of clinicopathologic characteristics of gastric follicular lymphomas and duodenal follicular lymphomas. Hum Pathol. 2017;65:201-8.

\section{Publisher's Note}

Springer Nature remains neutral with regard to jurisdictional claims in published maps and institutional affiliations.
Ready to submit your research? Choose BMC and benefit from:

- fast, convenient online submission

- thorough peer review by experienced researchers in your field

- rapid publication on acceptance

- support for research data, including large and complex data types

- gold Open Access which fosters wider collaboration and increased citations

- maximum visibility for your research: over 100M website views per year

At BMC, research is always in progress.

Learn more biomedcentral.com/submissions 